\title{
Effect of alumina-blasting pressure on adhesion of CAD/CAM resin block to dentin
}

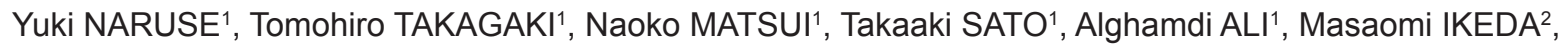 \\ Toru NIKAIDO ${ }^{1}$ and Junji TAGAMI ${ }^{1}$ \\ ${ }^{1}$ Cariology and Operative Dentistry Department, Graduate School of Medical and Dental Sciences, Tokyo Medical and Dental University, 1-5-45 \\ Yushima, Bunkyo-ku, Tokyo 113-8510, Japan \\ ${ }^{2}$ Oral Prosthetic Engineering, Graduate School of Medical and Dental Sciences, Tokyo Medical and Dental University, 1-5-45 Yushima, Bunkyo-ku, \\ Tokyo 113-8510, Japan \\ Corresponding author, Tomohiro TAKAGAKI; E-mail: takagaki.ope@tmd.ac.jp
}

\begin{abstract}
The aim of this study was to evaluate how alumina-blasting pressure affects the bond strength of CAD/CAM resin blocks (CRBs) to bovine dentin using two different types of resin cements. CRB slices were divided into three groups by alumina-blasting pressure, namely, untreated and $0.1 \mathrm{MPa}$ and $0.2 \mathrm{MPa}$, and further divided into three subgroups by combination of CRBs surface treatment and types of cement: Scotchbond Universal and RelyX ultimate (RXB), Scotchbond Universal and RelyX unicem2 (U2B), ceramic primer and RelyX unicem2 (U2C). The CRB slices were then cemented to bovine dentin, microtensile bond strength test was performed and evaluated. Regardless of the alumina-blasting pressure, RXB group have the highest $\mu$ TBS and bond strength tends to increase with increasing alumina-blasting pressure. Alumina-blasting to CRB surface by at an appropriate pressure and use of conventional resin cement were required to obtain strong adhesion with the tooth structure.
\end{abstract}

Keywords: CAD/CAM, $\mu$ TBS, Alumina-blast, Resin cement, Dentin

\section{INTRODUCTION}

Patient demands for esthetic dental-restoration and minimally invasive treatment-modes have recently made tooth-colored restorative materials a preferred choice. Computer-aided design and computer-aided manufacturing (CAD/CAM) technologies have enabled the production of dental-restoration products through numerically controlled machining, resulting in uniform material quality, greater reproducibility, and reduced production costs. CAD/CAM systems mainly use ceramic materials because of their esthetic qualities, surface finish, and long-term durability. Recently, CAD/CAM resin blocks (CRBs) have become available, thereby opening up avenues for a wider range of materials ${ }^{1}$. CRBs are innovative new CAD/CAM materials that provide superior esthetic results in easy steps. Three years of clinical trials have shown that resin-based composite CAD/CAM inlays work as well as porcelain $\mathrm{CAD} / \mathrm{CAM}$ inlays ${ }^{2)}$.

However, the mechanical properties of CRBs are inferior to those of ceramic blocks intended for the same applications. Thus, a major concern hindering the use of CRBs is their inferior long-term performance. Using industrial polymerization processes involving high temperatures and pressures, CAD/CAM systems can fabricate CRBs with higher degrees of conversion and lower residual monomers which is suitable for $\mathrm{CAD} /$ CAM processing ${ }^{3)}$. However, to improve the fracture resistance, ceramic restorations should be firmly bonded

Color figures can be viewed in the online issue, which is available at J-STAGE.

Received Jul 13, 2017: Accepted Dec 20, 2017

doi:10.4012/dmj.2017-237 JOI JST.JSTAGE/dmj/2017-237 to the underlying tooth substrate with an adhesive luting cement; therefore, these newly developed CRBs may face difficulties when bonding with resin cements ${ }^{4,5}$. Similarly, owing to their brittle nature, resin restorations should also be firmly bonded to the tooth substrate with an adhesive luting cement ${ }^{6}$.

A previous clinical survey of the results of $\mathrm{CAD} /$ CAM restoration revealed that approximately $10 \%$ of dental accounts reported that the crowns debonded at a rate higher than the industry standard (3M ESPE's Field Safety Corrective Action on June 15, 2015). However, little information is available about the cause of these debondings. Thus, an in vitro study is required to establish appropriate cementation protocols for $\mathrm{CAD} /$ CAM resin crown bonding to the tooth substrate.

One of the most common methods for conditioning the surface of polymeric materials prior to adhesion is using airborne-particle abrasion. Air abrasion with alumina particles cleans the surface and simultaneously increases the surface area ${ }^{7,8)}$; however, it can cause significant damage to the surfaces of the $\mathrm{CRBs}^{9}$. To address this issue, many in vitro studies have focused on the surface conditioning of $\mathrm{CRBs}^{9-12}$. However, most of these studies focused on the bonding between the CRBs. Reliable indirect restoration is clinically provided not only by proper bonding between the CRBs and cement but also by firm adhesion between the cement and the tooth substrate ${ }^{7,13,14)}$. However, limited information is available on the bonding between CRBs and dentin with different surface conditioning.

To address this issue, the present study evaluates how alumina-blasting pressure affects the bond strength 
of a CRB to bovine dentin with two different types of resin cements. The null hypotheses to be tested were: (a) alumina-blasting pressure would not affect the bond strength of a CRB to bovine dentin. (b) types of resin cement would not influence the bond strength of a CRB to bovine dentin.

\section{MATERIALS AND METHODS}

Materials used in this study

Table 1 lists the material compositions used in this study. We used a Lava Ultimate (Lava Ultimate, 3M ESPE, St.
Paul, MN, USA) as CRB and RelyX ultimate (RXU, 3M ESPE) as resin cement with tooth primer. Scotchbond Universal (SBU, 3M ESPE) was used as RXU tooth primer or as silane coupling agent for the CRBs. In addition, RelyX unicem2 (RU2, 3M ESPE) was used as a self-adhesive resin cement, and RelyX ceramic primer (CP, 3M ESPE) was used as silane coupling agent.

\section{Specimen preparation}

Figure 1 shows schematically how the specimens were prepared. Recently extracted bovine incisors, stored frozen, were used as test substrates. The labial surfaces of

Table 1 Composition of the materials used in the study

\begin{tabular}{|c|c|c|c|}
\hline Material & Manufacturer & Composition & Lot NO \\
\hline $\begin{array}{l}\text { Lava } \\
\text { Ultimate }\end{array}$ & 3M ESPE & $\begin{array}{l}\text { Bis-GMA, Bis-EMA, UDMA, TEGDMA, silica particles }(20 \mathrm{~nm}) \\
\text { zirconia particles }(4-11 \mathrm{~nm}) \text {, zirconia particles }(4-11 \mathrm{~nm}) \\
\text { nanoparticle clusters }(0.6-10 \mu \mathrm{m})\end{array}$ & 559450 \\
\hline $\begin{array}{l}\text { Rely X } \\
\text { Ultimate }\end{array}$ & 3M ESPE & $\begin{array}{l}\text { Base: Methacrylate monomers, radiopaque silanated fillers } \\
\text { initiator components, stabilizers, rheological additives Catalyst } \\
\text { paste: Methacrylate monomers radiopaque alkaline fillers initiator } \\
\text { components stabilizers, pigments, rheological additives fluorescence } \\
\text { dye dark polymerize activator for Scotchbond Universal }\end{array}$ & 555658 \\
\hline $\begin{array}{l}\text { Scotchbond } \\
\text { Universal }\end{array}$ & 3M ESPE & $\begin{array}{l}\text { Bis-GMA, 2-hydroxyethyl methacrylate decamethylene, } \\
\text { dimethacrylate, ethanol, water, silane treated silica, } \\
\text { 2-propenoic acid } 2 \text {-ethyl, reaction products with 1, 10-decanediol } \\
\text { and Phosphorous,oxide, copolymer of acrylic and itaconic acid, } \\
\text { camphorquinone,dimethlaminobenzoat }\end{array}$ & 557634 \\
\hline $\begin{array}{l}\text { Rely X } \\
\text { Unicem2 }\end{array}$ & 3M ESPE & $\begin{array}{l}\text { Base: Methacrylate monomers containing phosphoric acid groups } \\
\text { methacrylate monomers silanated fillers, Initiator components } \\
\text { stabilizers, rheological additives Catalyst paste Methacrylate } \\
\text { monomers components, stabilizers, rheological additives }\end{array}$ & 623453 \\
\hline $\begin{array}{l}\text { Rely X } \\
\text { Ceramic Primer }\end{array}$ & 3M ESPE & Ethyl, alcohol, water, methacryloxypropyltrimethoxysilane & N759704 \\
\hline K-etchant gel & Kuraray Noritake & $40 \%$ phosphoric acid, water & $5 \mathrm{M} 0024$ \\
\hline
\end{tabular}

Bis-GMA: bisphenol-A-glycidyl methacrylate, Bis-EMA: bisphenol-A- ethoxyl methacrylate, UDMA: urethane dimethacrylate, TEGDMA: triethyleneglycol dimethacrylate

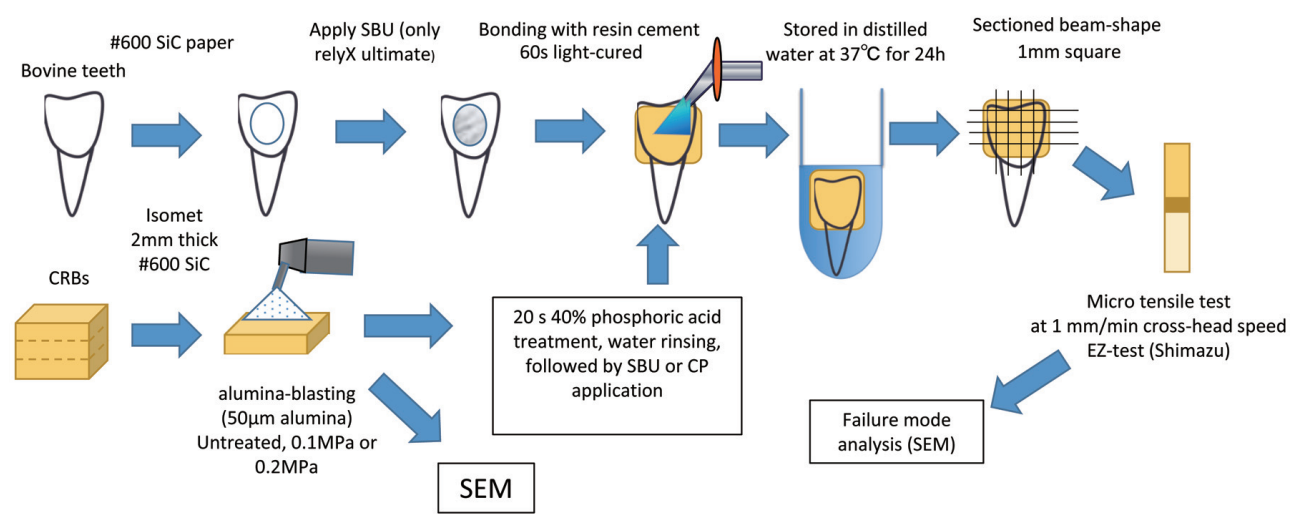

Fig. 1 Sample preparation for tensile bond test and SEM observation of adhesive interface. 
the teeth were ground with \#600-grit SiC paper (Sankyo Rikagaku, Okegawa, Japan) under running water to expose the dentin. The bovine teeth were sectioned in the middle of the crown by using a low-speed diamond-blade saw (Isomet, Buehler, Lake Bluff, IL, USA) under water irrigation, exposing areas of middle-depth dentin. The exposed dentin surfaces were wet ground by using \#600grit $\mathrm{SiC}$ paper to create flat surfaces with a standard smear-layer before cementation.

CRBs were cut into 45 slices with dimensions of approximately $15.5 \times 19.5 \times 2 \mathrm{~mm}$, by using the low-speed diamond-blade saw, followed by polishing with \#600-grit $\mathrm{SiC}$ paper. Prior to cementation, the CRB slices were divided into three groups: untreated and air-particle abrasion with $50-\mu \mathrm{m} \mathrm{Al}_{2} \mathrm{O}_{3}$ particles at 0.1 or $0.2 \mathrm{MPa}$ (10 s, $10 \mathrm{~mm}$ distance). Air abrasion was done by using a blasting machine (Basic Master, Renfert, Hilzingen, Germany). All CRB slices were cleaned with ethanol for 3 min in an ultrasonic bath.

\section{Scanning electron microscopy (SEM) prior to bonding} Alumina-sand blasted and \#600 polished CRB surface textures were examined by SEM (JSM-5310LV, JEOL, Tokyo, Japan) at $\times 350, \times 1,000$ and $\times 3,500$ magnification. For SEM observation, samples were mounted with carbon adhesion tape on a specimen holder and coated with gold and SEM observation was operated at $15 \mathrm{kV}$.

\section{Surface roughness analyses}

The surface roughness of specimens were measured using a laser scanning microscope (VK-X150, KEYENCE, Osaka, Japan) equipped with a $20 \times$ objective and each surface was measured 10 times. Quantification of the 3D surface roughness parameter Sa (arithmetic mean deviation) was performed and statistically compared using $t$-test.

\section{Bonding procedure}

For each group, the adhesive surface of 15 CRB slices was treated with phosphoric acid (K-etchant gel, Kuraray Noritake Dental, Tokyo, Japan) for $20 \mathrm{~s}$. After washing with water and air drying, $10 \mathrm{CRB}$ slices were treated with SBU and 5 CRB slices were treated with CP. Five CRB slices treated with SBU were then cemented to bovine dentin surfaces (polished by \#600 $\mathrm{SiC}$ paper) with RXU (RXB). Five CRB slices treated with SBU and 5 CRB slices treated with CP were cemented with RU2 (U2B and U2C). Cement layer was light-cured for $60 \mathrm{~s}$ by a halogen unit (Optilux 501, $600 \mathrm{~mW} / \mathrm{cm}^{2}$, Demetrom, Danbury, CT, USA). All specimens were then stored in distilled water for $24 \mathrm{~h}$, following which they were perpendicularly sectioned with the low-speed diamond saw at the dentin-adhesive interface into approximately $1 \times 1 \mathrm{~mm}$ beams. Five beams were obtained from each of the 5 CRB slices per material group. These specimens were then fixed to a universal testing machine (EZ-Test, Shimadzu, Kyoto, Japan) with a cyanoacrylate adhesive (Model Repair II Blue, Dentsply-Sankin, Tochigi, Japan) and subjected to microtensile bond strength ( $\mu$ TBS) testing at a crosshead speed of $1 \mathrm{~mm} / \mathrm{min}(n=25)$.

\section{Failure-mode analysis}

After debonding the specimens, failure modes were inspected by SEM imagery at $\times 100$ magnification and classified into one of the following five categories:

A: adhesive failure between CRB and resin cement;

$\mathrm{B}$ : cohesive failure within resin cement;

C: mixed failure, including adhesive failure between $\mathrm{CRB}$ and resin cement or between dentin and resin cement, cohesive failure within resin cement or within dentin;

D: adhesive failure between resin cement and dentin;

$\mathrm{E}$ : cohesive failure within dentin.

\section{Statistical analysis}

The difference between the mean value and standard deviation were decided by pilot study. Number of specimens was provided by sample size determination method for two tailed $t$-test as follows:

$$
\begin{aligned}
\mathrm{N} & =2\left(\mathrm{Z}_{\alpha / 2}+\mathrm{Z}_{\beta}\right)^{2} \mathrm{SD}^{2} / \Delta^{2} \\
& =2(1.96+1.282)^{2} 10^{2} / 10^{2}
\end{aligned}
$$$$
=21.02
$$

$\mathrm{Z}_{\alpha / 2}$ : Probability level=5\%, $\mathrm{Z}_{\beta}$ : Statistical power level $=90 \%$

SD: Standard deviation, $\Delta$ : The difference between the mean value

Number of specimens was set to 25 in each group. The $\mu$ TBS indicated normal distribution by Shapiro-Wilk test in each group $(p>0.05)$. Multiple comparisons between each group were made by using a two-way analysis of variance and $t$-test with Bonferroni's correction at a 95\% confidence level. All statistical procedure was carried out using SPSS ver. 22.0 (IBM, Chicago, IL, USA).

\section{RESULTS}

\section{SEM prior to bonding}

Figure 2 shows the SEM images of the CRB surface morphology. Many scratches caused by the \#600 SiC paper appear in the photomicrographs of CRBs without alumina blasting. At high magnification, small porosities below $5 \mu \mathrm{m}$ were also observed. After alumina blasting, numerous concave and convex features appear and scratches caused by the \#600 $\mathrm{SiC}$ paper disappear on the CRB surfaces. While roughening effects could be seen for both alumina-blasting pressure, no morphological difference could not be observed.

\section{Surface roughness analyses}

Table 2 shows the surface roughness of the CRB surface and Fig. 3 shows the laser scanning microscope 3D topography images of the specimen surface. The significant differences were observed between each group $(p<0.01)$, their surface roughness increased with an increase in the air pressure of alumina-blasting.

\section{Microtensile bond strength tests}

Table 3 gives the mean $\mu$ TBS values, standard deviations, and failure modes. Regardless of the alumina-blasting pressure, RXB group have the highest $\mu \mathrm{TBS}$, followed 


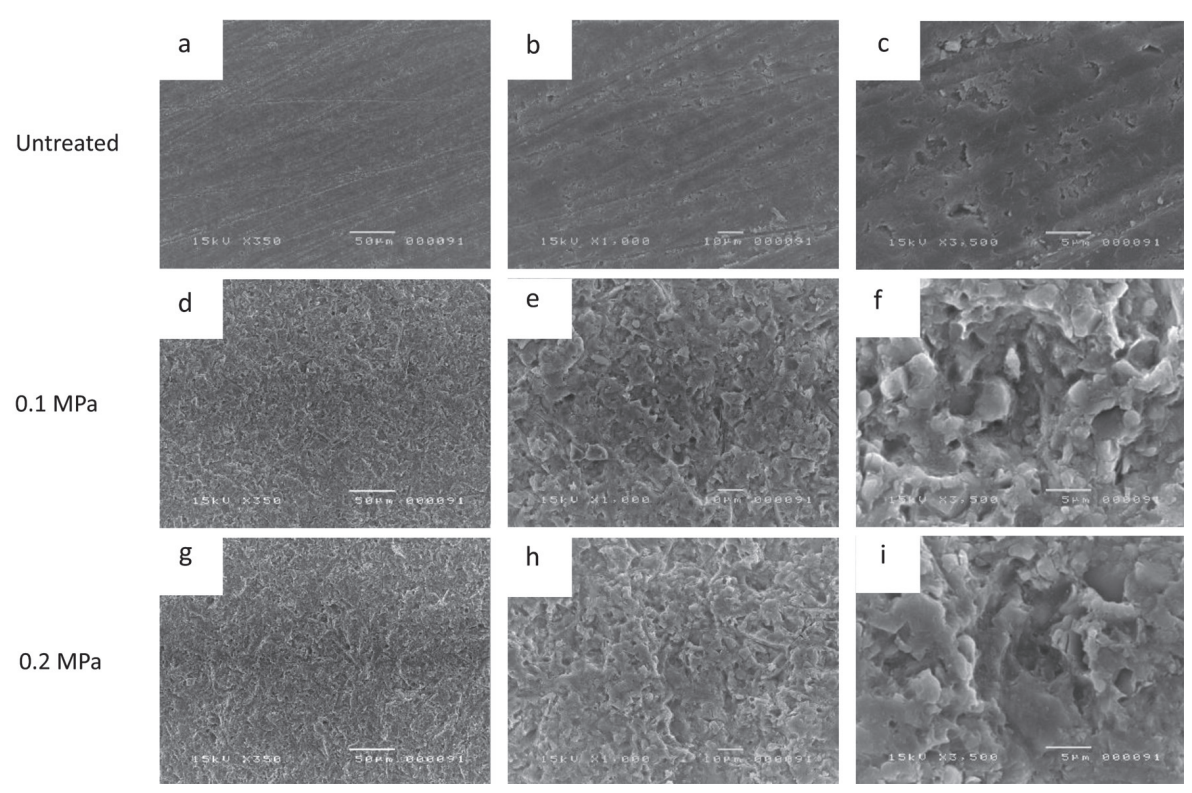

Fig. 2 Micrographs images of CRBs surface after alumina-blasting at different magnifications (a, d, g×350, b, e, h×1,000, c, f, i $\times 3,500)$.

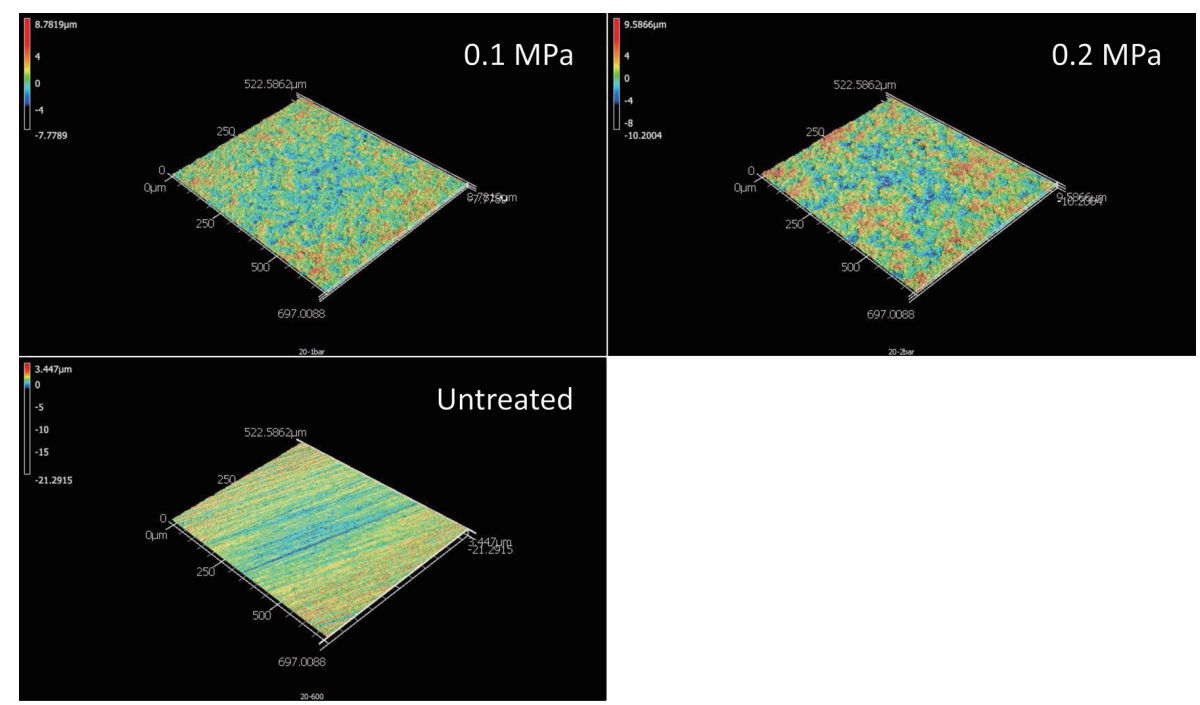

Fig. 3 Laser scanning microscope 3D topography images of the specimen surface showing untreated and alumina blasted (0.1 MPa, 0.2 MPa). All images are at the same scale. Many scratches caused by the \#600 SiC paper appear in the untreated group, their surface roughness increased with an increase in the air pressure of alumina-blasting.

Table 2 Surface roughness

\begin{tabular}{lc}
\hline & $\mathrm{Sa}(\mu \mathrm{m})$ \\
\hline Untreated & $0.68^{\mathrm{A}}(0.06)$ \\
$0.1 \mathrm{MPa}$ & $1.28^{\mathrm{B}}(0.13)$ \\
$0.2 \mathrm{MPa}$ & $1.98^{\mathrm{C}}(0.26)$ \\
\hline
\end{tabular}

Data are shown as mean (standard deviation).

Values with different superscript letters indicate statistically significant differences $(p<0.05)$. by the U2C and U2B groups. No significant difference occurs between RXB and U2C at untreated $(p=1.00)$, although a significant difference is found at untreated between each group except RXB and U2C $(p<0.01)$.

With RXB, no significant difference in bond strength occurs between alumina blasting at 0.1 and 0.2 $\mathrm{MPa}(p=1.00)$; however, the untreated group shows a significantly lower bond strength than either the 0.1 or $0.2 \mathrm{MPa}$ group $(p<0.01)$. Also, no significant difference appears between untreated and $0.1 \mathrm{MPa}$ in the RXB 
Table $3 \mu$ TBS values $(\mathrm{MPa})$ and types of failure mode

\begin{tabular}{|c|c|c|c|}
\hline & Untreated & $0.1 \mathrm{MPa}$ & $0.2 \mathrm{MPa}$ \\
\hline RXB & $\begin{array}{c}20.5^{\mathrm{A}}(5.39) \\
19 / 1 / 3 / 2 / 0\end{array}$ & $\begin{array}{c}52.6^{\text {b }}(6.09) \\
6 / 3 / 7 / 1 / 8\end{array}$ & $\begin{array}{c}52.0^{\mathrm{b}}(5.59) \\
0 / 4 / 5 / 1 / 15\end{array}$ \\
\hline $\mathrm{U} 2 \mathrm{~B}$ & $\begin{array}{c}8.96^{\mathrm{a}}(2.77) \\
10 / 0 / 0 / 15 / 0\end{array}$ & $\begin{array}{c}11.0^{\mathrm{a}}(3.54) \\
2 / 0 / 1 / 22 / 0\end{array}$ & $\begin{array}{c}22.0(3.30) \\
0 / 1 / 6 / 18 / 0\end{array}$ \\
\hline $\mathrm{U} 2 \mathrm{C}$ & $\begin{array}{c}19.4^{\mathrm{cA}}(2.93) \\
17 / 0 / 0 / 8 / 0\end{array}$ & $\begin{array}{c}20.8^{\mathrm{c}}(3.67) \\
0 / 0 / 3 / 22 / 0\end{array}$ & $\begin{array}{l}30.8(3.96) \\
0 / 0 / 10 / 15 / 0\end{array}$ \\
\hline
\end{tabular}

Number of specimens: 25

Data are shown as mean (standard deviation).

Values with same superscript letters were no statistically significant differences $(p>0.05)$.

Small letters show the horizontal significance and capital letters show the vertical significance for each group.

Numbers in square brackets show the number of specimens classified into five failure modes $[\mathrm{A} / \mathrm{B} / \mathrm{C} / \mathrm{D} / \mathrm{E}]$ :

(A): Adhesive failure between CRBs and cement. (B): Cohesive failure of the cement. (C): Mixed failure, including adhesive failure between CRB and resin cement or between dentin and resin cement, cohesive failure within resin cement or within dentin. (D): Adhesive failure between cement and dentin. (E) Cohesive failure of dentin.
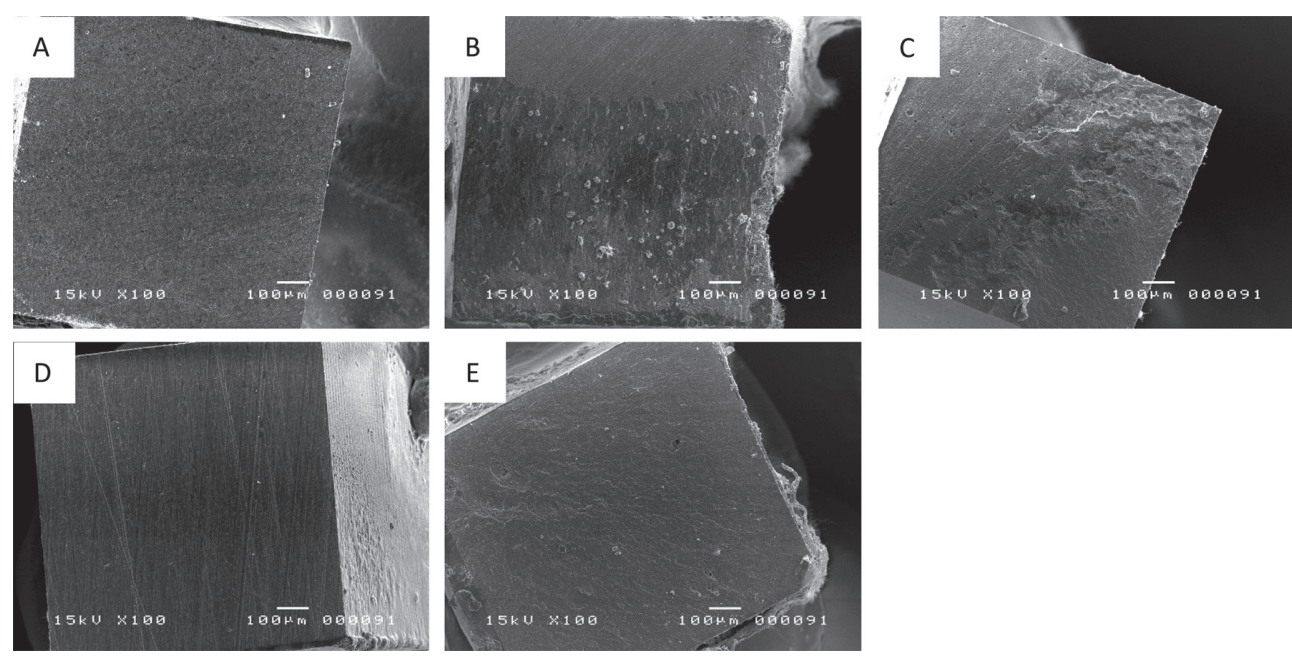

Fig. 4 Micrographs of the failure types, magnification of $100 \times$.

(A): Adhesive failure between CRBs and cement. (B): Cohesive failure of the cement. (C): Mixed failure, including adhesive failure between CRB and resin cement or between dentin and resin cement, cohesive failure within resin cement or within dentin. (D): Adhesive failure between cement and dentin. (E) Cohesive failure of dentin.

group ( $p=0.33$ ) and U2C group $(p=0.77)$, but the group alumina blasted at $0.2 \mathrm{MPa}$ shows a significantly large bond strength $(p<0.01)$. Overall, the bond strength tends to increase with increasing alumina-blasting pressure.

\section{Failure-mode analysis}

The distribution of the failure modes observed under a SEM was presented in Table 1. And Fig. 4 shows micrographs of the failure types, magnification of $100 \times$. In the U2B and U2C groups, adhesive failure between cement and dentin (type D) occurs regardless of the alumina-blasting pressure. As the alumina-blasting pressure increases, the adhesive failure between CRB and cement (type A) decreases, whereas the mixed failure (type C) and adhesive failure between cement and dentin (type D) increases. In the RXB group, significant adhesive failure appears between the CRBs and cement (type A) at untreated. As the alumina-blasting pressure increases, the adhesive failure between CRBs and cement (type A) decreases, whereas the cohesive failure of dentin (type E) increases. In all groups, the ratio of adhesive failure between the CRBs and cement (type A) tends to decrease as the alumina-blasting pressure increases. Adhesive failure between the CRBs and cement (type A) does not occur at $0.2 \mathrm{MPa}$. 


\section{DISCUSSION}

This study evaluates how alumina-blasting pressure affects the bond strength between CRBs and bovine dentin. Without alumina-blasting, free radicals were reported to be insufficient for achieving adhesion between the resin cements and CRB surfaces ${ }^{12)}$. The bond strength between CRBs and resin cement is improved by surface treatments such as mechanical treatment and the application of adhesive material. Strong bonding between CRBs and resin cement depends on micromechanical interlocking and chemical bonding to the surface of the restorative material, which in turn requires cleaning and roughening for adequate surface activation ${ }^{15)}$. However, previous studies indicate that alumina-blast flaws are similar in nature to true microcracks ${ }^{12,16-19)}$.

In the present study, alumina blasting improves the $\mu$ TBS in all groups and with increasing alumina-blasting pressure, the bond strength also tends to increase. SEM images of the CRB surfaces show no significant difference in the surface texture between alumina blasting at 0.1 and $0.2 \mathrm{MPa}$. However, measured with a laser scanning microscope, the surface roughness of 0.2 $\mathrm{MPa}$ are significantly higher than $0.1 \mathrm{MPa}$. The results of $\mu$ TBS and failure-mode analysis show that the $\mu$ TBS results at $0.2 \mathrm{MPa}$ are clearly greater than those at 0.1 $\mathrm{MPa}$. The failure-mode analysis indicates that the rate of adhesive failure between CRBs and resin cement (type A) decreases with increasing alumina-blasting pressure, and type-A failure disappears at $0.2 \mathrm{MPa}$. These results suggest that alumina-blasting pressure of $0.2 \mathrm{MPa}$ is a sufficient condition to obtain mechanical interlocking between the CRBs and resin cement. These results of this study reject of the null hypothesis that alumina-blasting pressure would not affect the bond strength of a CRB to bovine dentin. Since the failure-mode analysis does not reveal cohesive failure of the CRBs, the reduction of mechanical strength in the Lava Ultimate block because of the cracks on the block surface is not a problem until $0.2 \mathrm{MPa}$.

Recall that this study uses SBU or CP as the silane coupling agent. The results of $\mu$ TBS testing and the failure-mode analysis suggest that stable adhesion at untreated cannot be obtained by the silane coupling agent only via chemical bonding. The results of $\mu$ TBS in the U2C group show significantly higher bond strength than that in the U2B group. These results suggest that SBU is not effective or stable. Note that Kalavacharla et $a l$. reported that the silane constituent in the universal adhesive might not be effective in optimizing the resinceramic bond, so a separate silane treatment remains an inevitable step for glass-ceramic adhesion ${ }^{16)}$.

A silane functional monomer is activated upon reacting with water, thereby hydrolyzing to silanol, which adsorbs and chemically bonds to glass. However, upon hydrolysis silane may undergo dehydration condensation, thereby forming an oligomer that no longer bonds to glass ${ }^{20-22)}$. Silanol is reported to be stable in some water-alcohol concentrations at $\mathrm{pH} 4.6$. The
$\mathrm{pH}$ of Scotchbond Universal is 2.7; this low $\mathrm{pH}$ may promote hydrolysis and dehydration condensation ${ }^{22)}$. In the present study, the insufficient effectiveness of silane incorporated in SBU may have contributed to the higher incidence of CRB debonding.

The luting procedure is based on clinical adhesive strategies to bond the resin cement to tooth substrates in indirect restorations. Conventional adhesive cementation combines pretreatment of the tooth surface with a self-etch adhesive agent, followed by the application of a dual-polymerizing resin cement. The main difference between these adhesive systems is the tooth pretreatment with a primer containing a functional monomer. The effectiveness of the pretreatment of the tooth surface depends on the technique used to implement it and is also time consuming. The new selfadhesive, dual-polymerizing resin cements are designed to simplify such cementation procedures. Self-adhesiveresin cements contain acid monomers, resulting in an initial lower $\mathrm{pH}$ for the infiltration into the demineralized collagen network ${ }^{23,24)}$.

In the present study, significant differences appear in $\mu$ TBS between the conventional and self-adhesive resin cements. Regardless of alumina-blasting pressure, the lowest bond strength occurs for the U2B group. For the U2C group, no significant difference in the bond strength occurs with the RXB group without alumina blasting. However, after alumina blasting, the bond strength is significantly lower than for the RXB group, regardless of alumina-blasting pressure. Stawarczyk et al. reported a lower tensile bond strength of selfadhesive-resin composite cements to polymeric crowns compared with the bond strength of conventional selfetching resin cements ${ }^{9}$. The results of the failure-mode analysis suggest that adhesion to dentin of the selfadhesive resin cements is insufficient compared with the conventional type of resin cement. For the U2B and $\mathrm{U} 2 \mathrm{C}$ groups, the main failure modes are adhesive failure between cement and dentin (type D) and mixed failure (type C). Self-adhesive cement generally contains a significant quantity of phosphate functional monomer, which is reported to degrade the mechanical properties of the cement after curing ${ }^{11)}$. This is considered to be one of the factors leading to low bond strength. According to the results of this study, another null hypothesis that types of resin cement would not influence the bond strength of a CRB to bovine dentin is also rejected.

Clinically, resin cement is not expected to work without a priori alumina blasting, the absence of which leads to debonding at the resin-cement interface. Even with adequate surface roughness, self-adhesive cements result in a lower bonding performance because of their low dentin-bonding ability. To firmly bond CRBs to the underlying tooth substrate, we thus recommend using conventional resin cement with separate and dedicated primers and alumina blasting at $0.2 \mathrm{MPa}$ on CRBs.

One limitation of the present study that should be acknowledged is that only a single kind of resin block, conventional resin cement and self-adhesive resin cement were evaluated. Further studies should clarify whether 
sandblasting are also effective surface treatments with other CRBs and other resin cements.

\section{CONCLUSION}

To obtain stable adhesion, we suggest treatment of the CRB surface by alumina blasting at an appropriate pressure. Furthermore, the use of conventional resin cement may be required to obtain strong adhesion with the tooth structure.

\section{REFERENCES}

1) Park SH, Yoo YJ, Shin YJ, Cho BH, Baek SH. Marginal and internal fit of nano-composite CAD/CAM restorations. Restor Dent Endod 2016; 41: 37-43.

2) Fasbinder DJ, Dennison JB, Heys DR, Lampe K. The clinical performance of CAD/CAM-generated composite inlays. J Am Dent Assoc 2005; 136: 1714-1723.

3) Nguyen JF, Migonney V, Ruse ND, Sadoun M. Resin composite blocks via high-pressure high-temperature polymerization. Dent Mater 2012; 28: 529-534

4) Burke FJ, Wilson NH, Watts DC. Fracture resistance of teeth restored with indirect composite resins: the effect of alternative luting procedures. Quintessence Int 1994; 25: 269-275.

5) Scherrer SS, de Rijk WG, Belser UC, Mayer JM. Effect of cement film thickness on the fracture resistance of a machinable glass-ceramic. Dent Mater 1994; 10: 172-177.

6) Burke FJ. The effect of variations in bonding procedure on fracture resistance of dentin-bonded all-ceramic crowns. Quintessence Int 1995; 26: 293-300.

7) Furukawa K, Inai N, Tagami J. The effects of luting resin bond to dentin on the strength of dentin supported by indirect resin composite. Dent Mater 2002; 18: 136-142.

8) Marshall SJ, Bayne SC, Baier R, Tomsia AP, Marshall GW. A review of adhesion science. Dent Mater 2010; 26: e11-e16.

9) Stawarczyk B, Basler T, Ender A, Roos M, Ozcan M, Hämmerle C. Effect of surface conditioning with air borneparticle abrasion on the tensile strength of polymeric CAD/ CAM crowns luted with self-adhesive and conventional resin cements. J Prosthet Dent 2012; 107: 94-101.

10) D'Arcangelo C, Vanini L. Effect of three surface treatments on the adhesive properties of indirect composite restorations. J Adhes Dent 2007; 9: 319-326.

11) Higashi M, Matsumoto M, Kawaguchi A, Miura J, Minamino T, Kabetani T, Takeshige F, Mine A, Yatani H. Bonding effectiveness of self-adhesive and conventional-type adhesive resin cements to CAD/CAM resin blocks. Part 1: Effects of sandblasting and silanization. Dent Mater J 2016; 35: 21-28.

12) Nobuaki A, Keiichi Y, Takashi S. Effects of air abrasion with alumina or glass beads on surface characteristics of CAD/ CAM composite materials and the bond strength of resin cements. J Appl Oral Sci 2015; 23: 629-636.

13) Fuentes MV, Escribano N, Ceballos L. Effect of indirect composite treatment microtensile bond strength of selfadhesive resin cements. J Clin Exp Dent 2016; 1; 8: e14-21.

14) Swift EJ Jr, Brodeur C, Cvitko E, Pires JA. Treatment of composite surfaces for indirect bonding. Dent Mater 1992; 8: 193-196.

15) Park JH, Choi YS. Microtensile bond strength and micromorphologic analysis of surface-treated resin nanoceramics. J Adv Prosthodont 2016; 8: 275-284.

16) Kalavacharla VK, Lawson NC, Ramp LC, Burgess JO. Influence of etching protocol and silane treatment with a universal adhesive on lithium disilicate bond strength. Oper Dent 2015; 40: 372-378.

17) Kosmac T, Oblak C, Jevnikar P, Funduk N, Marion L. The effect of surface grinding and sandblasting on flexural strength and reliability of Y-TZP zirconia ceramic. Dent Mater 1999; 15: 426-433.

18) Kosmac T, Oblak C, Jevnikar P, Funduk N, Marion L. Strength and reliability of surface treated Y-TZP dental ceramics. J Biomed Mater Res 2000; 53: 304-313.

19) Zhang Y, Lawn BR, Rekow ED, Thompson VP. Effect of sandblasting on the long-term performance of dental ceramics. J Biomed Mater Res 2004; 71: 381-386.

20) Lung CY, Matinlinna JP. Aspects of silane coupling agents and surface conditioning in dentistry: an overview. Dent Mater 2012; 28: 467-477.

21) Matinlinna JP, Vallittu PK. Bonding of resin composites to etchable ceramic surfaces-an insight review of the chemical aspects on surface conditioning. J Oral Rehabil 2007; 34: 622630.

22) Yoshihara K, Nagaoka N, Sonoda A, Maruo Y, Makita Y, Okihara T, Irie M, Yoshida Y, Van Meerbeek B. Effectiveness and stability of silane coupling agent incorporated in 'universal' adhesives. Dent Mater 2016; 32: 1218-1225.

23) Behr M, Rosentritt M, Regnet T, Lang R, Handel G. Marginal adaptation in dentin of a self-adhesive universal resin cement compared with well-tried systems. Dent Mater 2004; 20: 191197.

24) Poggio C, Pigozzo M, Ceci M, Scribante A, Beltrami R, Chiesa M. Influence of different luting protocols on shear bond strength of computer aided design/computer aided manufacturing resin nanoceramic material to dentin. Dent Res J (Isfahan) 2016; 13: 91-97. 